
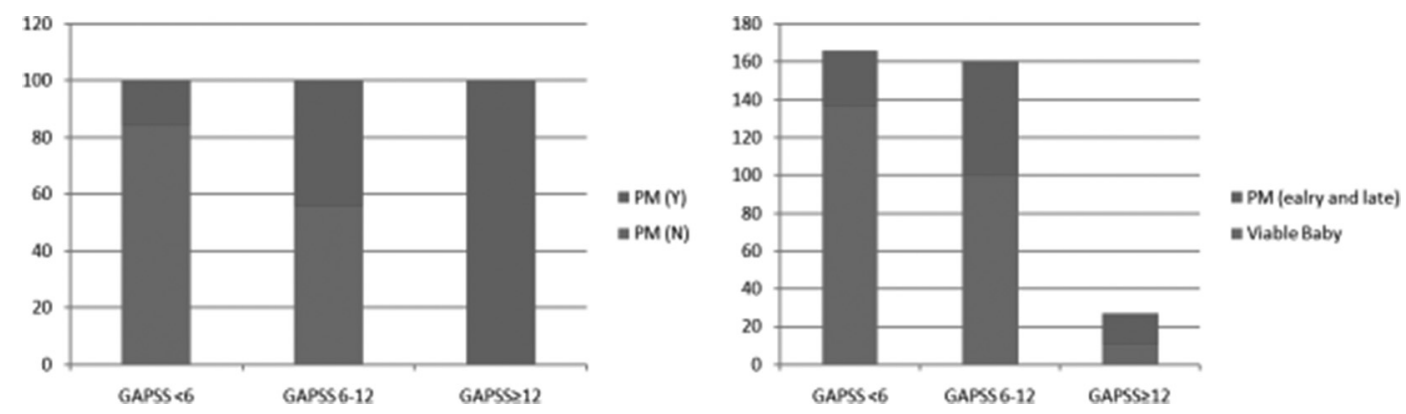

Abstract 59 Figure 1 Pregnancy morbidity and live births divided in the three groups

risk group (GAPSS 6-11, $\mathrm{n}=66$ ) and a high risk group (GAPSS12, n=5).

When considering patients who ever experienced PM while treated with SoC, all patients in the high risk group experienced PM, while patients in the medium group had a significant higher rate of PM when compared to the low risk group [29 (43.9\%) patients V.s. 11 (15.3\%), respectively; $\mathrm{p}<0.001]$. When analysing the number of pregnancies in the three groups, patients in the high risk group had significantly lower live birth rates, when compared to the other groups [11 (40.7\%) life births V.s. 100 (62.1\%) and 137 (82.5\%), respectively; $\mathrm{p}<0.05]$. Furthermore, patients with medium risk group also had significantly lower live birth rates, when compared to the lower risk group $(\mathrm{p}<0.001)$.

Figure 1 resumes the results of PM and live births divided in the three groups.

Conclusions GAPSS might be a valuable tool in identifying patients at higher risk of developing any event of PM who might need additional therapeutic approach other than SoC.

Funding Source(s): N/A

\section{ASSOCIATION OF SYSTEMIC LUPUS ERYTHEMATOSUS (SLE) GENETIC SUSCEPTIBILITY LOCI WITH LUPUS NEPHRITIS IN CHILDHOOD-ONSET AND ADULT-ONSET WITH SLE}

\begin{abstract}
${ }^{1}$ Declan Webber, ${ }^{1}$ Jingjing Cao, 'Daniela Dominguez, ${ }^{2}$ Dafna D Gladman, ${ }^{3}$ Deborah Levy, ${ }^{1}$ Lawrence $\mathrm{Ng},{ }^{4}$ Andrew Paterson, ${ }^{2}$ Zahi Touma, ${ }^{2}$ Murray B Urowitz, ${ }^{5}$ Joan Wither, ${ }^{1}$ Earl D Silverman, 'Linda T Hiraki*. ${ }^{1}$ The Hospital for Sick Children; ${ }^{2}$ Krembil Research Institute, University of Toronto; ${ }^{3}$ Hospital for Sick Children and University of Toronto; ${ }^{4}$ Sickkids Hosp; ${ }^{5}$ University of Toronto
\end{abstract}

\subsection{6/lupus-2019-Ism.60}

Background Lupus nephritis (LN) is one of the most common and severe manifestations of systemic lupus erythematosus (SLE). We tested the association of SLE-risk loci with LN risk in childhood- (cSLE) and adult-onset SLE (aSLE).

Methods Two Toronto-based tertiary care SLE cohorts included cSLE (diagnosed <18 y) and aSLE patients (diagnosed 18y). Patients met ACR and/or SLICC SLE criteria and were genotyped on the Illumina MEGA or Omni1 arrays. Ungenotyped SNPs were imputed (1000 Genomes Project). HLA alleles were imputed in the Europeans only (SNP2HLA). Ancestry was inferred using principal components. We identified those with and without biopsy confirmed LN. HLA and non-HLA additive SLE risk-weighted genetic risk scores (GRSs) were tested for association with LN risk in logistic models, stratified by cSLE/aSLE and ancestry. Stratified effect estimates were meta-analyzed.
Results Of 1237 participants, 572 had cSLE (41\% with LN) and 665 had aSLE (30\% with LN). Increasing non-HLA GRS was significantly associated with increased LN risk $(\mathrm{OR}=1.26 ; 95 \% \mathrm{CI}: 1.09,1.46, \mathrm{p}=0.0006)$ as was increasing HLA GRS in Europeans $(\mathrm{OR}=1.55 ; 95 \% \mathrm{CI}: 1.07,2.25$; $\mathrm{p}=0.03$ ). There was a trend for stronger associations between both GRSs and LN risk in Europeans with cSLE compared with aSLE.

Conclusions We observed an association between known SLErisk loci and LN risk in children and adults with SLE, with the strongest effect observed among Europeans with cSLE. Future directions will include incorporating SLE-risk SNPs specific to non-European ancestral groups and validating findings in an independent cohort.

Funding Source(s): Dr. Hiraki: Canadian Institute of Health Research (CIHR) Project Scheme grant

\section{DIFFERENCES IN ORGAN SYSTEM INVOLVEMENT ACROSS RACIAL/ETHNIC GROUPS: RESULTS FROM THE CALIFORNIA LUPUS EPIDEMIOLOGY STUDY}

Stephanie Rush*, Laura Trupin, Patricia Katz, Maria C DallEra, Jinoos Yazdany. UC San Francisco

\subsection{6/lupus-2019-Ism.61}

Background SLE is a chronic disease that affects many organ systems and can cause permanent damage. We sought to determine if there are differences in patterns of SLE organ system damage among racial/ethnic groups.

Methods Data derive from the baseline visit of the California Lupus Epidemiology Study (CLUES), an ongoing cohort of patients in the San Francisco Bay Area with confirmed SLE diagnoses. Participants provided access to medical records and had a visit with a study rheumatologist. Race/ ethnicity (White, African American, Hispanic of any race, and Asian) was determined by patient report. Due to the small sample size, patients from other racial groups were excluded from this analysis $(n=5)$. Disease damage was measured using the SLICC/ACR Damage Index (SDI), calculated at the study visit. We examined damage at the organ system level, using items from the SDI, and defined glucocorticoid-related damage as avascular necrosis, diabetes, cataracts, and osteoporosis. Logistic regression was used to estimate the prevalence of damage in each organ system and the prevalence of glucocorticoid-related damage by race/ethnicity controlling for current age. We ranked the prevalence of damage in each organ system that comprises the SDI for each racial/ethnic group. 
Abstract 61 Table 1 SLICC Damage index (SDI) by race/ethnicity

\begin{tabular}{|c|c|c|c|c|c|c|c|c|c|c|c|}
\hline \multirow{3}{*}{ SDI, mean (sd) } & \multicolumn{2}{|c|}{ Total $(n=323)$} & \multicolumn{2}{|c|}{ Asian $(n=125)$} & \multicolumn{2}{|c|}{ Non-Hispanic Black $(n=34)$} & \multicolumn{2}{|c|}{ Hispanic $(n=69)$} & \multicolumn{2}{|c|}{ Non-Hispanic White $(\mathrm{n}=95)$} & \multirow{2}{*}{$\begin{array}{c}\text { p-value } \\
0.394\end{array}$} \\
\hline & \multicolumn{2}{|c|}{$1.8(0.4)$} & \multicolumn{2}{|c|}{$1.8(0.2)$} & \multicolumn{2}{|c|}{$2.2(0.3)$} & \multicolumn{2}{|c|}{$2.0(0.2)$} & \multicolumn{2}{|c|}{$1.6(0.2)$} & \\
\hline & $\%$ & rank & $\%$ & rank & $\%$ & rank & $\%$ & rank & $\%$ & rank & \\
\hline Musculoskeletal & $24 \%$ & 1 & $21 \%$ & 2 & $24 \%$ & 2 & $24 \%$ & 2 & $29 \%$ & 1 & 0.648 \\
\hline Ocular & $21 \%$ & 2 & $23 \%$ & 1 & $19 \%$ & 3 & $27 \%$ & 1 & $17 \%$ & 3 & 0.398 \\
\hline Neuropsychiatric & $19 \%$ & 3 & $16 \%$ & 3 & $18 \%$ & 4 & $13 \%$ & 6 & $25 \%$ & 2 & 0.265 \\
\hline Skin & $14 \%$ & 4 & $11 \%$ & 6 & $31 \%$ & 1 & $14 \%$ & 4 & $10 \%$ & 5 & 0.021 \\
\hline Renal & $14 \%$ & 5 & $16 \%$ & 4 & $17 \%$ & 5 & $21 \%$ & 3 & $7 \%$ & 6 & 0.087 \\
\hline Vascular & $10 \%$ & 6 & $12 \%$ & 5 & $11 \%$ & 8 & $14 \%$ & 5 & $6 \%$ & 7 & 0.415 \\
\hline Malignancy & $8 \%$ & 7 & $3 \%$ & 11 & $8 \%$ & 9 & $7 \%$ & 8 & $14 \%$ & 4 & 0.060 \\
\hline Glucocorticoid-related damage & $28 \%$ & & $30 \%$ & & $37 \%$ & & $30 \%$ & & $22 \%$ & & 0.195 \\
\hline
\end{tabular}

Results Among 323 participants, 89\% were female, 39\% Asian, 11\% African American, 22\% Hispanic of any race, and 29\% White. Mean age was $45 \pm 14$; mean age at diagnosis $29 \pm 12$. SDI ranged from 0 to 10 points, mean $1.8 \pm 2.0$; $70 \%$ of the cohort had SDI $>0$. There were no differences in mean SDI by race/ethnicity $(p=0.4$; see Table). Musculoskeletal and ocular damage were among the five most common systems for all groups. African Americans were significantly $(p=0.02)$ more likely to have skin damage than the other racial/ethnic groups. Non-Hispanic whites were the only group that had malignancy in their top five systems of SDI damage. Renal damage was most prevalent among Hispanics and least prevalent among whites. Glucocorticoid-related damage was more prevalent among African Americans in comparison to whites, although this difference was not statistically significant.

Conclusions There are differences in prevalence of damage by organ system among racial/ethnic groups, with blacks significantly more likely to have skin manifestations and whites more likely to have a history of cancer. There is also evidence of higher glucocorticoid-related damage among blacks. Further research is required to explain what leads to these differences; they could be related to quality and access to care and treatment, or to differential disease biology or environmental exposures.

Funding Source(s): CDC (U01 DP005120)

\section{RACIAL DIFFERENCES IN SELF-EFFICACY AND PATIENT- PROVIDER INTERACTIONS AMONG PATIENTS WITH SYSTEMIC LUPUS ERYTHEMATOSUS}

Kai Sun*, Amanda M Eudy, Jennifer L Rogers, Lisa G Criscione-Schreiber, Jayanth Doss, Rebecca E Sadun, Megan EB Clowse. Duke University

\subsection{6/lupus-2019-Ism.62}

Background Significant racial disparities exist in the prevalence and outcomes of systemic lupus erythematosus (SLE). Few studies have examined modifiable factors intrinsic to a patient encounter that may contribute to such disparities. We aimed to explore potential areas for intervention to reduce racial disparities with a focus on patient self-efficacy and the quality of patient-provider interactions.
Methods Cross-sectional data were collected from consecutive SLE patients actively treated and followed at a tertiary lupus clinic. Patient-provider interaction was measured using the Interpersonal Processes of Care survey (IPC-29), which has 7 domains on a 5 -point Likert scale including Hurried communication, Elicited concerns, Explained results, Patientcentered decision making, Compassionate respectful, Discrimination, and Disrespectful office staff. General self-efficacy, self-efficacy for managing medications and treatments, and patient-reported health status were measured using Patient-Reported Outcomes Measurement Information System (PROMIS) short forms. Additional demographic and clinical information were gathered by survey and chart review. SLE Disease Activity Index (SLEDAI), Systemic Lupus International Collaborating Clinics (SLICC) Damage scores, and Medication Regimen Complexity Index were calculated. Bivariate analyses compared patient characteristics of Caucasians and non-Caucasians.

Results 84 enrolled (37\% Caucasians, 59\% African American, $1 \%$ Native American, and 4\% Hispanic). Non-Caucasians compared to Caucasians are younger, more likely to be single/unmarried, on disability, and less likely to have college education. Non-Caucasians tend to report less fatigue, better social health, carry a more recent SLE diagnosis but take a more complex rheumatic medication regimen, have higher SLEDAI, lower fibromyalgia symptom severity, and a trend for higher damage scores (table 1). In terms of modifiable factors intrinsic to the patient encounter, there were no significant differences in patient-provider interactions except that non-Caucasians rated providers to have more Hurried communication. Non-Caucasians also had a trend for lower general self-efficacy.

Conclusions Non-Caucasians come from more disadvantaged sociodemographic backgrounds and have worse SLE disease outcomes. Overall, scores for patient-rated interactions with physicians in this selective sample were better than in other studies. However, non-Caucasians reported more Hurried communication with their providers and lower general self-efficacy. These may be modifiable factors within a patient encounter to reduce healthcare disparities in SLE. Whether quality of patient-provider communication and patient self-efficacy predict higher disease activity and damage scores longitudinally should be investigated. 